\title{
A BRIEF NOTE ON COHERENT FEEDBACK IN SEMICONDUCTOR LASERS
}

\author{
M.A. GRADO CAFFARO, consultant (Madrid, Spain) \\ and M. GRADO CAFFARO, consultant (Madrid, Spain) \\ C./Julio Palacios, $11,9^{\circ} \mathrm{B}, 28029-M a d r i d$ (Spain) \\ (Received August 12, 1993; in final form September 10, 1993)
}

Some aspects on coherent feedback in a laser diode are investigated. In particular, weak optical feedback is considered in the context of the feedback-induced frequency shift. In addition, the Lang-Kobayashi equations are considered.

\section{INTRODUCTION}

The main problems related to the physics of laser diodes with optical feedback are not completely solved, particularly with respect to theoretical aspects. Some experimental work has been performed recently. ${ }^{1,2}$ For example, experimental observations on relaxation oscillation with optical feedback have been realized. ${ }^{1}$ Theoretical treatments have also been proposed. ${ }^{1,2}$ By using more powerful methods based on fundamental physics, it is feasible to describe theoretically the phenomena associated with the relaxation oscillation mentioned above.

In the following, we shall study laser diodes with optical feedback and in the absence of optical feedback. In particular, the minimum value of the current that satisfies the condition $\mathrm{P}_{\mathrm{sp}} \ll \mathrm{P}_{\mathrm{st}}$ will be considered $\left(\mathrm{P}_{\mathrm{sp}}\right.$ and $\mathrm{P}_{\mathrm{st}}$ are the densities of spontaneous and stimulated emitted photons, respectively). Furthermore, weak optical feedback will be analyzed.

\section{THEORY}

First, we formulate the well-known Lang-Kobayashi equations, namely: $:^{3,4}$

$$
\begin{aligned}
& \frac{d}{d t}[E(t)]=\frac{1}{2}\left[G(n(t))-\Gamma_{0}\right] E(t)+\gamma E(t-\tau) \exp \left(-j \omega_{0} \tau\right) \\
& \frac{d}{d t}[n(t)]=-T^{-1} n(t)-G_{A}(n(t)) P(t)+\frac{J}{e d}(j=\sqrt{-1})
\end{aligned}
$$

where $E(t)$ is the slowly varying envelope of the complex electric field in the active layer of the laser diode, $G$ is the complex gain function that depends on the density 
of electron-hole pairs $n(t), \Gamma_{0}$ is the rate of power emission from the laser, $\gamma$ is the feedback rate, $\tau$ is the feedback delay-time, and $\omega_{0}$ is the optical angular frequency that is close to the laser angular frequency. In addition, $T$ is the life-time for spontaneous recombination of electrons and holes in the active layer, $P(t)=|E(t)|^{2}$ is the density of photons in the active layer, $J$ is the pump current density, $d$ is the thickness of the active layer, and $e$ is the electronic charge. In particular, $G$ is given by: ${ }^{4.5}$

$\mathrm{G} \equiv \mathrm{G}_{\mathrm{A}}+\mathrm{jG}_{\mathrm{D}}=\Gamma_{0}+\zeta(1+\mathrm{j} \alpha)\left[\mathrm{n}(\mathrm{t})-\mathrm{n}_{0}\right]$

where $G_{A}$ is the absorptive part of the gain and $G_{D}$ is the dispersive part; $\zeta$ is the gain coefficient, $\alpha$ is the linewidth-enhancement parameter, and $n_{0}$ is the electronhole density without feedback.

From eq. (3) it is deduced:

$\mathrm{G}_{\mathrm{A}}(\mathrm{t})=\Gamma_{0}+\left[\mathrm{n}(\mathrm{t})-\mathrm{n}_{0}\right] \zeta$

$\mathrm{G}_{\mathrm{D}}(\mathrm{t})=\zeta \alpha\left[\mathrm{n}(\mathrm{t})-\mathrm{n}_{0}\right]$

By substituting eq. (4) into eq. (2) we get:

$J(t)=\operatorname{ed}\left[\dot{n}(t)+\left(T^{-1}+\zeta P_{s}\right) n(t)+\left(\Gamma_{0}-\zeta n_{0}\right) P_{s}\right]$

where the dot denotes derivative with respect to time. We have considered harmonic solutions of eq. (1) in the form $E_{s}(t)=P_{s}^{1 / 2} \cdot \exp (j \Omega t)$ where $\Omega$ is the so-called feedback-induced frequency shift that is given by the following expression namely: ${ }^{4.5}$

$\Omega=\gamma(\sec \phi) \sin \left[\phi-\left(\omega_{0}+\Omega\right) \tau\right]$

with $\phi=\tan ^{-1} \alpha$.

This shift is related to both AM and FM noises that are very important in the context of coherent feedback. Optical feedback produces a competition between the two exterior modes involved. In particular, the FM noise is due to the switching of the frequency at random values when the diode mode is near the center between the mentioned modes. This noise acts in conjunction with the AM noise.

On the other hand, for weak optical feedback we can assume that $\Omega \ll \omega_{0}$. Thus, eq. (7) reduces to:

$\Omega=\gamma(\sec \phi) \sin \left(\phi-\omega_{0} \tau\right)$

with both $\Omega$ and $\gamma$ very small.

Finally, we shall consider the minimum pump current responsible for the preceding noise phenomena. This current can be regarded approximately as the current involved in the following equation: ${ }^{4}$

$\mathrm{P}_{\mathrm{st}(\min )}=\frac{\mathrm{P}_{\mathrm{sp}}\left(1+\alpha^{2}\right) \Gamma_{0} \mathbf{i}_{\min }}{2(\delta \omega) \mathrm{i}}$ 
where $\mathrm{P}_{\mathrm{sp}} \ll \mathrm{P}_{\mathrm{st}}$ and $\delta \omega$ stands for the full width at half-maximum around the laser angular frequency; in other words, $\delta \omega$ is the bandwidth corresponding to the absence of optical feedback so that the semiconductor laser emits monochromatic optical radiation.

\section{CONCLUSION}

We have investigated several aspects of the semiconductor lasers in the context of optical feedback. In particular, the Lang-Kobayashi equations have been used; these equations play an important role and they can be considered as the Langevin equations in accordance with the work of C.H. Henry. ${ }^{6}$

In addition, we emphasize the relevance of eqs. (6), (8) and (9) in order to understand the mechanisms associated with both AM and FM noises. These phenomena have been investigated recently by M.A. Grado and M. Grado. ${ }^{4}$

\section{REFERENCES}

1. J.S. Cohen, R.R. Drenten, B.H. Verbeek. IEEE J. Quantum Electron., 24 (1988), 1989.

2. B.H. Verbeek, D. Lenstra, A.J. den Boef. Philips Tech. Rev. 43 (1987), 292.

3. R. Lang, K. Kobayashi. IEEE J. Quantum Electron., 16 (1980), 347.

4. M.A. Grado, M. Grado, unpublished.

5. G.A. Acket, D. Lenstra, A.J. den Boef, B.H. Verbeek. IEEE J. Quantum Electron., 20 (1984), 1163.

6. C.H. Henry. IEEE J. Quantum Electron., 19 (1983), 1391. 



Submit your manuscripts at

http://www.hindawi.com
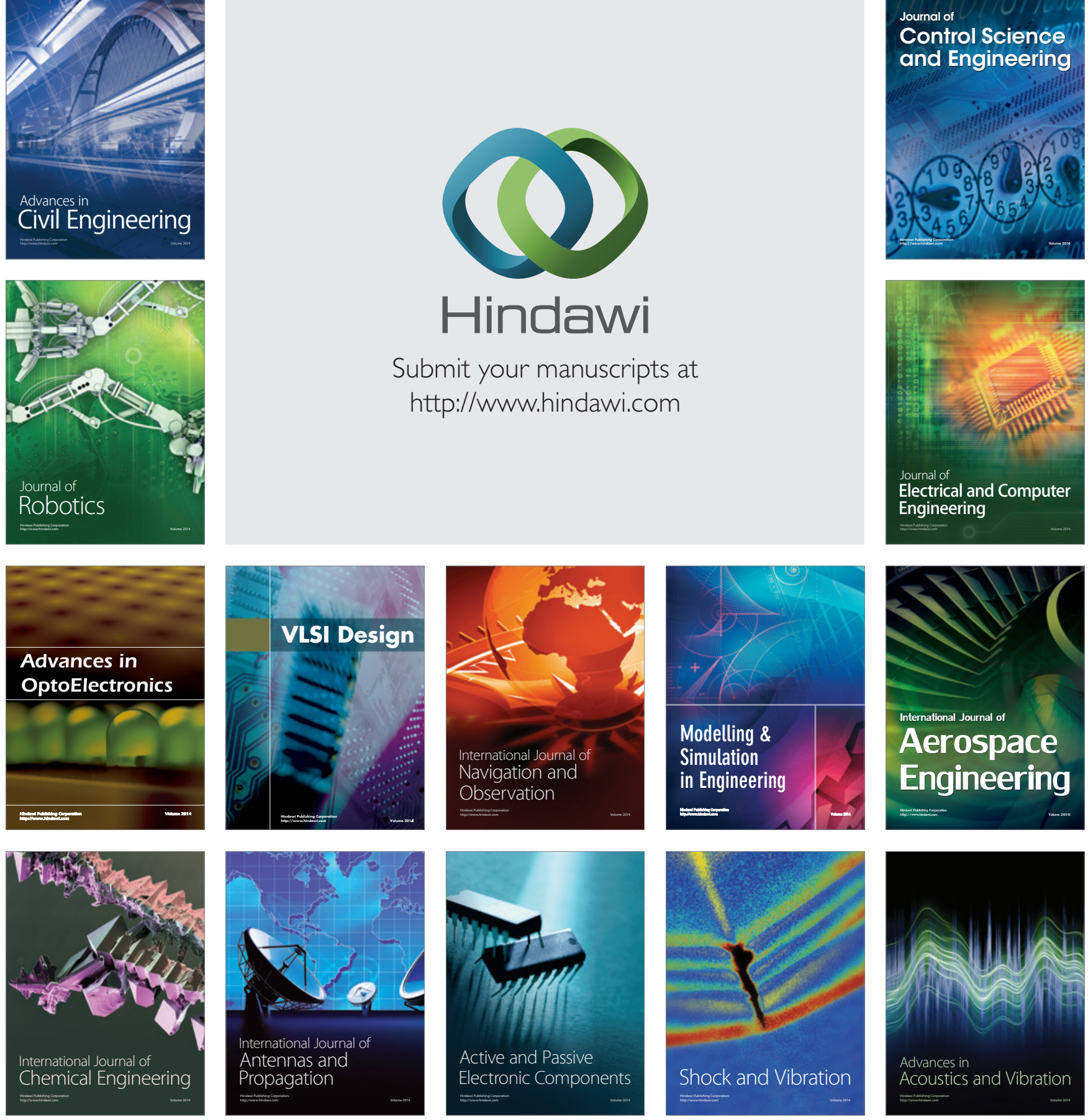